\title{
Risk analysis of the re-emergence of Plasmodium vivax malaria in Japan using a stochastic transmission model
}

\author{
Tomoyuki Bitoh · Kaoru Fueda • Hiroshi Ohmae • \\ Mamoru Watanabe $\cdot$ Hirofumi Ishikawa
}

Received: 31 May 2010/Accepted: 29 September 2010/Published online: 26 October 2010

(c) The Japanese Society for Hygiene 2010

\begin{abstract}
Objectives This study analyzed the risk of infection with Plasmodium vivax in local residents through a stochastic simulation in which an infected tourist, local resident, or immigrants from an endemic area would visit Himi-shi, Toyama prefecture, which is a formerly endemic area in Japan.

Methods In Toyama, the habitats of Anopheles sinensis, which can transmit $P$. vivax, have been examined previously. We constructed a stochastic model of $P$. vivax transmission that can handle small numbers of infected persons and infected mosquitoes. The seasonal fluctuation in the numbers of captured An. sinensis was taken into account in the model.

Results Ten thousand trial simulations were carried out stochastically with a range of human blood indexes (HBI) of $1-10 \%$ for a range of months (June-September). The simulation results for a realistic assumption of a $1 \% \mathrm{HBI}$ showed that the risk of infection for local residents was low (below 1\%) except for the immigrants scenario.

Conclusions The risk of infection among local residents (second cycle) was estimated to be very low for
\end{abstract}

T. Bitoh $\cdot$ K. Fueda $\cdot$ H. Ishikawa $(\bowtie)$

Department of Human Ecology,

Graduate School of Environmental Science,

Okayama University, Okayama 700-8530, Japan

e-mail: ishikawa@ems.okayama-u.ac.jp

H. Ohmae

Department of Parasitology,

National Institute of Infectious Diseases,

Tokyo 162-8640, Japan

M. Watanabe

Department of Medical Entomology,

National Institute of Infectious Diseases,

Tokyo 162-8640, Japan all situations. Therefore, there is little possibility for $P$. vivax infection to become established in this area of Japan.

Keywords Re-emergence Plasmodium vivax . Toyama . Anopheles sinensis · Stochastic simulation

\section{Introduction}

This study analyzed the risk of infection with Plasmodium vivax for local residents through a stochastic simulation in which an infected tourist, local resident, or immigrants from an endemic area would visit Himi-shi, Toyama prefecture, a formely endemic area in Japan.

Before World War II, the inhabitants of the central part of the main island of Japan, especially Aichi, Toyama, Ishikawa, Fukui, and Shiga prefectures, suffered from $P$. vivax malaria [1]. After World War II, the prevalence of $P$. vivax was attributed to indigenous malaria and imported malaria derived from demobilized soldiers and citizens repatriated from overseas [2]. Recently, there have been no reports of malaria infection in Japan, except for imported malaria. About 61-109 patients with imported malaria, including 21-39 P. vivax patients, were reported in Japan between 2000 and 2006 [3].

The Toyama Institute of Health [4] has issued reports on the habitats of Anopheles sinensis-which is recognized as a $P$. vivax malaria vector-in Toyama prefecture, and there have also been observations for Anopheles mosquitoes in Shiga prefecture [5].

There have been several reports of the local transmission of $P$. vivax imported by a patient; for example, in Singapore [6]; far north Queensland, Australia [7]; Corsica, France [8]; and Michigan, United States [9]. 
Global warming may lead to increased numbers of malaria-vector mosquitoes and expansion of their habitats [10]. In South Korea, indigenous $P$. vivax malaria disappeared in the latter half of the $1970 \mathrm{~s}$, but $P$. vivax infection has been maintained since its re-emergence was first detected in 1993 [11-13]. The re-emergence of $P$. vivax was believed to originate from mosquitoes infected with $P$. vivax in North Korea, which subsequently moved to South Korea [14].

Most mathematical models of $P$. vivax transmission have adopted deterministic systems. De Zoysa et al. [15] examined the relapse of hypnozoites, and Ishikawa et al. [16] proposed a transmission model developed from the Dietz-Molineaux-Thomas model [17] to analyze prevalence in the Solomon Islands. Fujita et al. [18] investigated the relapse pattern of hypnozoites in South Korea. Ponçon et al. [19] assessed spatial variations in the risk of $P$. falciparum and $P$. vivax re-emergence in a formerly endemic region of southern France, adopting a probability distribution approach, and Nakagawa et al. [20] analyzed the risk of the re-emergence of $P$. falciparum on Ishigaki island, Japan, in the future, employing a stochastic model.

Our model of transmission for $P$. vivax handled infected mosquitoes and persons stochastically. The model took into account the seasonal fluctuation in the number of captured An. sinensis. Because the number of infected persons and also the number of mosquitoes would be small in the targeted area, unlike the numbers in an endemic area, stochastic simulations were regarded as adequate to estimate the risk of infection with $P$. vivax in the targeted area.

In the model we used the human blood index (HBI), a malariological parameter that expresses the proportion of human blood meals to blood-fed female mosquitoes. We designed scenarios with a 1-day-stay tourist, a local resident returning from abroad, or immigrants washed ashore who were infected with $P$. vivax visiting the targeted area during June-September, and the HBI of An. sinensis as set as $1-10 \%$. The simulations showed that the risk of infection among local residents [second cycle (SC)] was estimated to be very low for all situations. Therefore, there was little possibility of the establishment of $P$. vivax infection among them.

\section{Materials and methods}

Study area

\section{Seasonal fluctuation in the number of captured An. sinensis mosquitoes}

In this study, Himi-shi, Toyama prefecture, was chosen as the study area. Himi-shi had a population of 54,945 in 2005 [21]. The Toyama Institute of Health [4] conducted capture

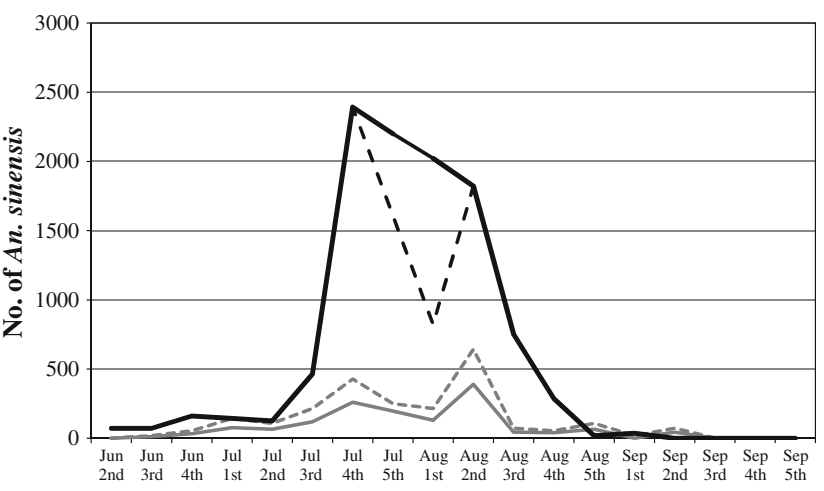

Fig. 1 Numbers of Anopheles sinensis captured using a light-trap in Himi-shi, Toyama. The gray-solid, black-broken, and gray-broken lines indicate 1993, 1994, and 1995 observations, respectively, and the black-solid line indicates modification of the 1994 observations

surveillance of mosquitoes, using light-traps in stockyards at more than ten spots in Toyama prefecture between 1969 and 2004, in which the number of captured An. sinensis was reported. We used the 1994 data of An. sinensis in Himi-shi from the viewpoint of risk analysis, because the highest density was observed in 1994 during the period of surveillance 1980-1996. An. sinensis first appeared in the 2nd week of June, and the number captured increased from the 3rd week of July, reached a peak in the 4 th week of July, and decreased thereafter. In the model we modified the number captured between the 4 th week of July and the 2nd week of August using a straight line to avoid a sharp fluctuation because a drop of unknown cause was observed in the 1st week of August (Fig. 1).

\section{Frequency of mosquito bites}

The mean frequency of bites by An. sinensis was assumed to be $0-3$ per human per night in proportion to the number of captured An. sinensis, and the frequency was assumed to be governed by a Poisson distribution. The gonotrophic cycle was fixed at 3 days [22].

\section{Period of sporozoite formation}

The periods of sporozoite formation for $P$. vivax were estimated to be $16-17$ and $9-10$ days at 20 and $25^{\circ} \mathrm{C}$, respectively [23]. In this study, the average period of sporozoite formation was assumed to depend on the temperature in Himi-shi, and the periods were assumed to be governed by a gamma distribution, with a mean of 9-17 days and a variance of $2^{2}$ (range 7-19 days).

Daily survival rate of An. sinensis

The daily survival rate of An. sinensis was assumed to be governed by a Bernoulli distribution, with means of 71, 82, 87, and $79 \%$ in June, July, August, and September, 
respectively; this assumption was based on surveillance in South Korea in 1999-2000 [24].

Epidemiological parameters

\section{Relapse}

Relapses occur frequently in $P$. vivax infection, and the relapse period varies widely $[25,26]$. In this study, the relapse period was classified into two patterns: short and long relapse periods, of 1-4 months and more than 5 months, respectively [18]. The proportion of primary infections to relapses and the proportion of the short to long relapse patterns were assumed to be governed by a Bernoulli distribution, with means of 20:80 and 34:66, respectively [26], where the short and long relapse periods were assumed to be governed by an exponential distribution with a mean of 52 days and a variance of $52^{2}$ and a log-normal distribution with a mean of 293 days and a variance of $59^{2}$, respectively [18].

\section{Incubation period of primary infection}

The incubation period of the primary infection with $P$. vivax was estimated to be 8 days [25], so it was assumed to be governed by a gamma distribution with a mean of 8 days and a variance of $2^{2}$.

\section{Acquisition rate of infectivity and period for loss of infectivity}

The period for loss of infectivity was assumed to be governed by a gamma distribution with a mean of 19 days and a variance of $8.3^{2}$; these values were derived from a maximum likelihood estimation from the observation of the transition to loss of infectivity in patients with gametocytes when chloroquine was administered[27]. Gametocytes were detected in 615 (28.9\%) of 2,125 P. vivax-infected persons in Thailand (1993-2003) [28]. The acquisition rate of infectivity per day was assumed to be governed by a Bernoulli distribution with a mean of $2.8 \%$ so as to assimilate the ratio of a PG-subclass in the positive class (for definition of "PG", see below "Model scheme") at 28.9\%.

\section{Diagnostic period}

The diagnostic period was assumed to be governed by a gamma distribution with a mean of 20 days and a variance of $5^{2}$.

\section{Model scheme}

A stochastic model was constructed for $P$. vivax transmission among humans and mosquitoes. The human population was divided into five epidemiological classes: negative, negative with hypnozoite (RE), incubation (PI), positive, and diagnosis. The positive class was subdivided into two subclasses: positive and infectious subclass with gametocytes (PG) and positive but not infectious subclass without gametocytes (PN). The mosquito population was divided into three epidemiological classes: negative, incubation, and positive.

Transfers among the epidemiological classes proceeded stochastically following the probability distributions. A local resident in the negative class who was bitten by vectors in the positive class was transferred to the RE- or PI-class. A resident would be transferred from the PI-class to the PN-subclass after the incubation period. A resident or an index patient in the PN- and PG-subclasses would be transferred between the PN- and PG-subclasses until diagnosis. An individual who was transferred from the PN- or PG-subclass to the diagnosis class would receive medical treatment, and afterwards he or she would be transferred to the negative class. A resident in the RE-class would be transferred to the positive class after relapse.

An. sinensis vectors that bite an individual in the PGsubclass were treated singly and stochastically in the model regarding daily survival, the period of sporozoite formation, and injection into residents. The model scheme for human-mosquito transmission of $P$. vivax is shown in Fig. 2, and the configuration of stochastic distributions in the model is summarized in Table 1 . When the value of the probability distribution exceeds the range in Table 1, the stochastic process replaces it with the lower or upper limit of its range.

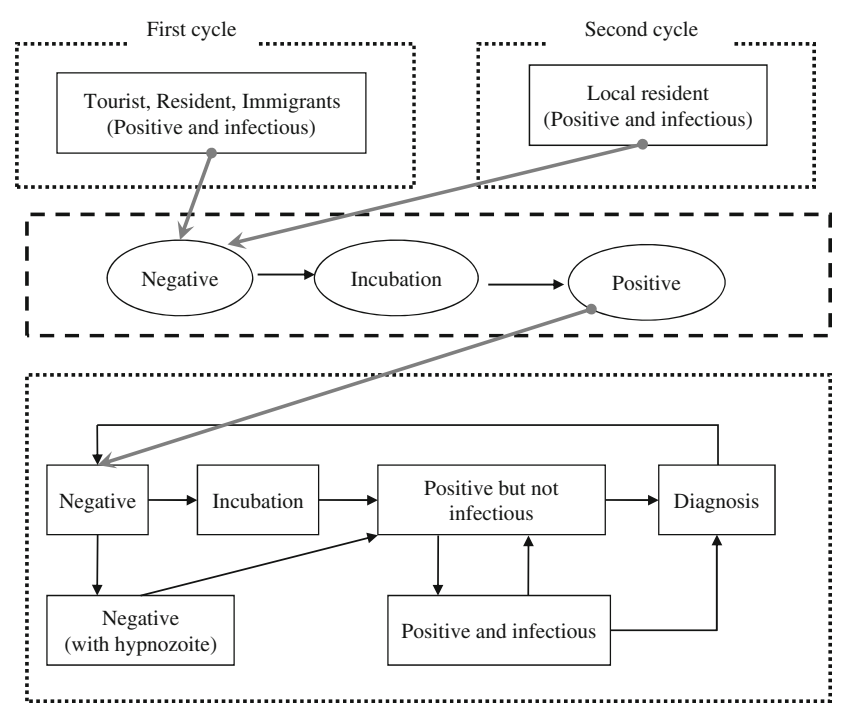

Fig. 2 Model schemes for Plasmodium vivax transmission of infection in the first and second cycles. Dotted and dashed rectangles indicate the profiles of human and vector stages, respectively. Ovals and solid rectangles indicate the profiles of vector classes and human classes, respectively 
Table 1 Configuration of the stochastic distribution for invasion

\begin{tabular}{|c|c|c|c|c|c|c|}
\hline Description & \multicolumn{2}{|c|}{$\begin{array}{l}\text { Type of } \\
\text { distribution }\end{array}$} & Mean & Variance & \multicolumn{2}{|l|}{ Range } \\
\hline \multicolumn{7}{|l|}{ Assumed values } \\
\hline Frequency of mosquito bites & \multicolumn{2}{|c|}{ Poisson } & $\begin{array}{l}0-3 \text { bites/human/night (depending } \\
\text { on Anopheles sinensis density) }\end{array}$ & - & \multicolumn{2}{|c|}{ June-September } \\
\hline Diagnostic period & \multicolumn{2}{|c|}{ Gamma } & 20 (days) & $5^{2}\left(\right.$ day $\left.^{2}\right)$ & \multicolumn{2}{|l|}{ 15-25 (days) } \\
\hline Description & & $\begin{array}{l}\text { Type of } \\
\text { distribution }\end{array}$ & Mean & Variance & Range (days) & References \\
\hline \multicolumn{7}{|l|}{ Estimated values } \\
\hline Period of sporozoite formation & & Gamma & 9-17 (days) (depending on temperature) & $2^{2}\left(\right.$ day $\left.^{2}\right)$ & $7-19$ & [23] \\
\hline Daily survival rate of mosquitoes & & Bernoulli & 71-87 (\%) (depending on season) & - & - & {$[24]$} \\
\hline Ratio of primary infection & & Bernoulli & Primary: $20(\%)$ (relapse: $80(\%))$ & - & - & {$[26]$} \\
\hline Incubation period in primary infectio & & Gamma & 8 (days) & $2^{2}\left(\mathrm{day}^{2}\right)$ & $6-10$ & [25] \\
\hline Relapse pattern of hypnozoites & & Bernoulli & Short: 34 (\%) (long: $66(\%))$ & - & - & {$[18]$} \\
\hline Relapse period (short) & & Exponential & 52 (days) & $52^{2}\left(\right.$ day $\left.^{2}\right)$ & $30-120$ & [18] \\
\hline Relapse period (long) & & Log-normal & 293 (days) & $59^{2}\left(\right.$ day $\left.^{2}\right)$ & $120-510$ & [18] \\
\hline Rate of gametocyte acquisition per d & day & Bernoulli & 2.8 (\%/days) & - & - & [28] \\
\hline Loss of infectivity & & Gamma & 19 (days) & $8.3^{2}\left(\mathrm{day}^{2}\right)$ & $0-40$ & {$[27]$} \\
\hline
\end{tabular}

In the model, local residents were assumed to have no immunity to $P$. vivax. We used first cycle (FC) or SC according to the case where the infection of a local resident is caused by an index patient or the infection is caused by an infected resident.

\section{Scenarios for invasions}

We investigated three cases of $P$. vivax malaria patient(s) who would visit the targeted area during June-September; (1) a 1-day-stay tourist who exhibited gametocytemia during their stay, (2) a local resident returning from abroad who was infected with $P$. vivax and developed parasitemia after returning home, and (3) ten immigrants washed ashore who were infected with $P$. vivax and belonged to the positive class when landing and stayed in the area for 10 days. For cases (2) and (3), a patient was regarded as having gametocytes or not according to the probability distribution. We also investigated three HBI levels of $A n$. sinensis assigned as 1, 5, and 10\% (Table 2).

\section{Results}

Ten thousand trial simulations were executed for 1,000 days following the index case scenarios (Table 2) where the time step of a stochastic process was adopted as a day. The range of risk of infection with $P$. vivax among local residents during June-September with a $1 \%$ HBI was evaluated as $0.01-0.94,0.01-0.34$, and $0.11-1.61 \%$ for the index case(s) of a tourist, a resident returning from abroad,
Table 2 Scenarios for invasions

\begin{tabular}{lllll}
\hline Index case(s) & & & HBI \\
Attribution & $\begin{array}{l}\text { Number of } \\
\text { patient(s) }\end{array}$ & $\begin{array}{l}\text { The length } \\
\text { of stay (day(s)) }\end{array}$ & $\begin{array}{l}\text { Visiting } \\
\text { months }\end{array}$ & \\
\hline Tourist & 1 & 1 & June-September & 1 \\
Resident & 1 & - & & 5 \\
Immigrants & 10 & 10 & & 10 \\
\hline
\end{tabular}

$H B I$ human blood index

and immigrants, respectively. The risk of SC with $P$. vivax infection spreading among local residents for an assumption of a high HBI of $10 \%$ was estimated to be very low, at 0.52 and $0.34 \%$ at most, for a 1-day-stay tourist (1) and a local resident returning from abroad (2), respectively, while the risk of SC was estimated to be slightly higher, at $1.73 \%$ at most, for the immigrant index cases (3). The simulation results are summarized in Table 3 .

\section{Discussion}

In the model in the present study, we evaluated the risk of infection with $P$. vivax for local residents by applying a stochastic process to infected mosquitoes in addition to individuals.

Because the process of gametocyte production is uncertain, the acquisition rate of infectivity was decided by malariological surveillance $[27,28]$. The distribution of the diagnostic period was taken from reports from Korea (1995) [29]. 


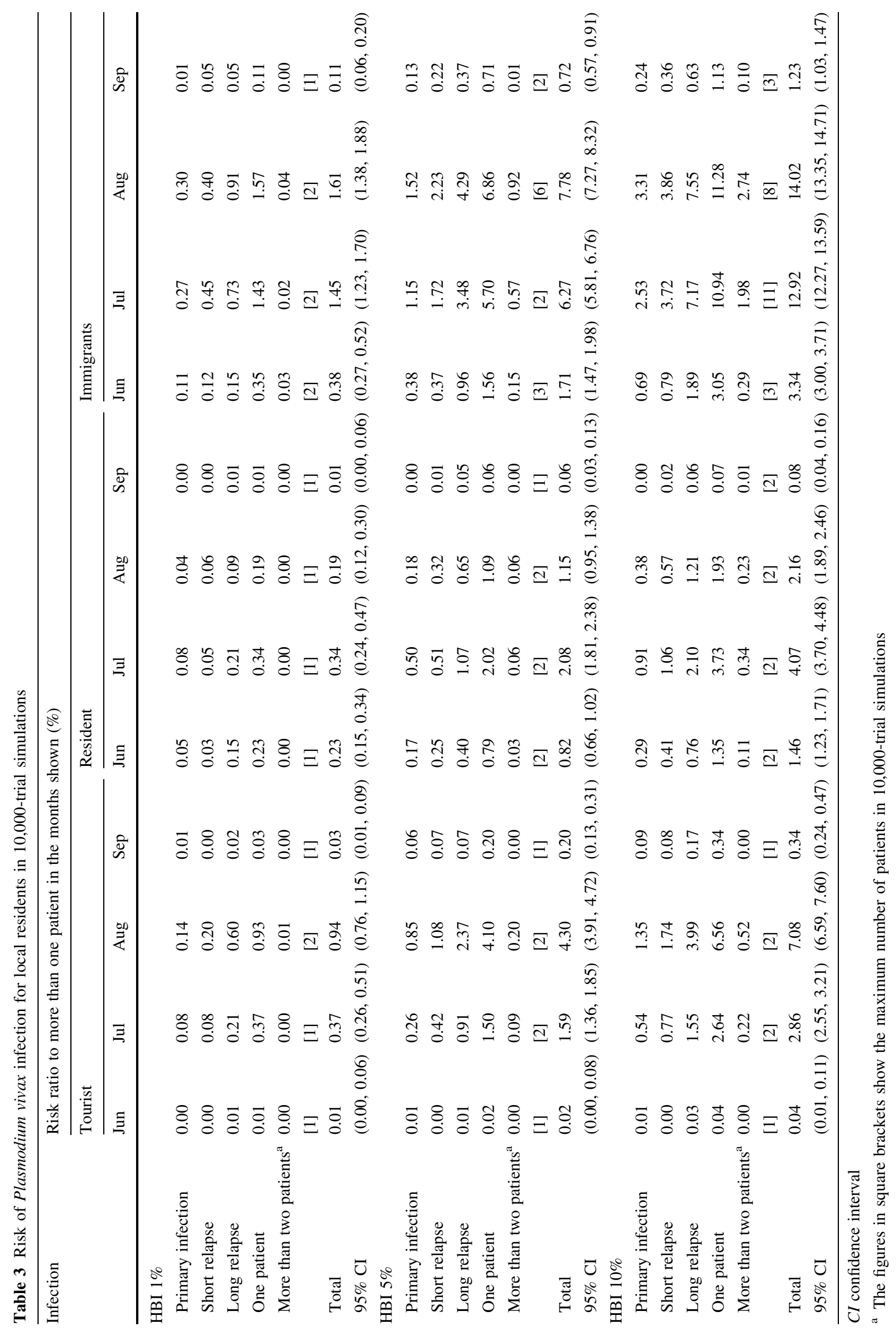


An. sinensis has a marked preference for animal blood meals [30], but simulations were carried out employing three levels of $\operatorname{HBI}(1,5$, and $10 \%)$ from the viewpoint of risk analysis. The risk of infection among local residents also depends on the density of An. sinensis. The observations of mosquitoes in Himi-shi, Toyama, which were collected employing light-traps in a stockyard, revealed a low density (Fig. 1). The number of mosquitoes collected using light-traps in a stockyard was about 360-fold higher than the number of mosquitoes collected using dry icetraps in the open air in the observation of Anopheles mosquitoes along the eastern shore of Lake Biwa, a formerly endemic area of $P$. vivax in Japan, on August 1, 2009 (unpublished work, M. Watanabe). The simulations were limited to low An. sinensis density.

The simulation results showed that August and July had higher risks of infection than other months for a visiting 1-day-stay tourist (1) and a local resident returning from abroad (2) as an index patient, respectively, and that both July and August would have almost the same level of risk of infection from immigrants (3); these simulation results were derived from the different assumptions on gametocytemia.

In Australia in 2002, a man with imported P. vivax malaria visiting a camping ground caused an outbreak, and 10 persons were infected with $P$. vivax due to the large number of $A n$. farauti, which have a marked capacity to transmit malaria [31]; this finding is in contrast to the situation in Toyama, with a low density of An. sinensis, but there were no SC patients in that Australian outbreak [7]. In the present model, the risk of infection among local residents was estimated to be low (below 1\%), and, in addition, there was only one patient involved in most cases of $P$. vivax infection, based on the scenario of a tourist (2) with the realistic assumption of a $1 \%$ HBI with regard to highly zoophilic An. sinensis. However, the risk would increase to about $4-7 \%$ if malaria vectors had a high HBI (5-10\%), such as An. culicifacies [31], equivalent to the somewhat high-level nature of $P$. vivax transmission (Table 3).

Infected persons with $P$. vivax coming from endemic countries are sometimes asymptomatic or have no typical symptoms. Consequently, a delay in the diagnosis increases the possibility of expanding infections in the vicinity of the infected person [32, 33]. However, the model in the present study was limited to patients with symptoms, especially regarding the situation of immigrants.

In the United States, about 300 patients with imported $P$. vivax malaria were reported in 2003, more than $40 \%$ of whom were infected in Asia [34]. In Taiwan, about 40 patients with imported malaria, including $20 \mathrm{P}$. vivax patients, were reported every year during the period 1991-2000 [35], and in Japan, about 20-40 patients with imported $P$. vivax malaria were reported in 2001-2006 [3].
Although the density of $A n$. sinensis has been reduced since 1997 [4], global warming may increase the numbers of $A n$. sinensis and expand their habitats. Therefore, it is desirable to carry out surveillance for An. sinensis continuously.

Acknowledgments We are indebted to the Toyama Institute of Health for providing the data on mosquitoes captured in Toyama prefecture. This work was supported in part by a Grant-in-Aid from the Ministry of Health, Labour and Welfare of Japan (H20-Sinkouippan-015), a Grant-in-Aid from the Japan Society for the Promotion of Science (21540129), and a Grant-in-Aid from the Ministry of the Environment (S-4, S-8 to H. O.).

\section{References}

1. Otsuru M. Malaria studies in Japan after World War II. Part I. Epidemiological aspects of postwar malaria. Jpn J Exp Med. 1959;30:23-32.

2. Tanaka S, Sugita S, Marui E. Two types of malaria epidemics during the postwar occupation period in Japan. Nippon Eiseigaku Zasshi 2009;64:3-13 (article in Japanese with English abstract).

3. National Institute of Infectious Diseases. Infectious Disease Surveillance Center. Malaria, 1999-2005. Infect Agents Surveill Rep. 2007;28:1-3 (in Japanese). http://idsc.nih.go.jp/iasr/28/323/ tpc323-j.html.

4. Toyama Institute of Health. Japanese encephalitis epidemic predicted surveillance for thirty-five years in Toyama. In: Nagai Y, Watanabe $\mathbf{M}$, editors. Toyama: Toyama Institute of Public Health; 2004 (article in Japanese).

5. Public Health and Science Center in Shiga. Surveillance of West Nile virus vector mosquito, 2008 (in Japanese). http://www.pref. shiga.jp/e/ef45/kansen-c/file/2008-WNV.pdf.

6. Lee YC, Tang CS, Ang LW, Han HK, James L, Goh KT. Epidemiological characteristics of imported and locally-acquired malaria in Singapore. Ann Acad Med Singap. 2009;8:40-9.

7. Hanna JN, Ritchie SA, Eisen DP, Cooper RD, Brookes DL, Montgomery BL. An outbreak of Plasmodium vivax malaria in Far North Queensland, 2002. Med J Aust. 2004;180:24-8.

8. Armengaud A, Legros F, D'Ortenzio E, Quatresous I, Barre H, Houze S, et al. A case of autochthonous Plasmodium vivax malaria, Corsica, August 2006. Travel Med Infect Dis. 2008;6: $36-40$.

9. Sunstrum J, Elliott LJ, Barat LM, Walker ED, Zucker JR. Probable autochthonous Plasmodium vivax malaria transmission in Michigan: case report and epidemiological investigation. Am J Trop Med Hyg. 2001;65:949-53.

10. Rogers DJ, Randolph SE. The global spread of malaria in a future, warmer world. Science. 2000;289:1763-6.

11. Paik YH, Ree HI, Shim JC. Malaria in Korea. Kyung Hee Univ Med J. 1987;12:17-31.

12. Chai JY. Re-emerging Plasmodium vivax malaria in the Republic of Korea. Korean J Parasitol. 1999;37:129-43.

13. Yeom J, Ryu SH, Oh S, Lee WJ, Kim TS, Kim KH, et al. Status of Plasmodium vivax malaria in the Republic of Korea during 2001-2003. Am J Trop Med Hyg. 2005;73:604-8.

14. Kho WG, Jang JY, Hong ST, Lee HW, Lee WJ, Lee JS. Border malaria characters of reemerging vivax malaria in the Republic of Korea. Korean J Parasitol. 1999;37:71-6.

15. De Zoysa AP, Mendis C, Gamage-Mendis AC, Weerasinghe S, Herath PRJ, Mendis KN. A mathematical model for Plasmodium vivax malaria transmission: estimation of the impact of transmission-blocking immunity in an endemic area. Bull World Health Organ. 1991;69:725-34. 
16. Ishikawa H, Ishii A, Nagai N, Ohmae H, Harada M, Suguri S, et al. A mathematical model for the transmission of Plasmodium vivax malaria. Parasitol Int. 2003;52:81-93.

17. Dietz K, Molineaux L, Thomas A. A malaria model tested in the African savannah. Bull World Health Organ. 1974;50:347-57.

18. Fujita K, Chen TT, Nishina T, Ishikawa H. Modeling of reemerging Plasmodium vivax in the north area of the Republic of Korea based on a mathematical model. J Fac Environ Sci Technol Okayama Univ. 2006;11:1-7.

19. Ponçon N, Tran A, Toty C, Luty AJ, Fontenille D. A quantitative risk assessment approach for mosquito-borne diseases: malaria re-emergence in southern France. Malar J. 2008;7:147.

20. Nakagawa Y, Ueki M, Fueda K, Ohmae H, Ishikawa H. Risk assessment of re-emerging Plasmodium falciparum on Ishigaki Island using a stochastic transmission model. Trop Med Health. 2009;37:97-107.

21. Ministry of Internal Affairs and Communications Bureau of Statistics of Japan. National census, 2005 (in Japanese). http://www. city.himi.toyama.jp/ct/other000004400/0120-100406-913.pdf.

22. Ree HI. Studies on Anopheles sinensis, the vector species of vivax malaria in Korea. Korean J Parasitol. 2005;43:75-92.

23. World Health Organization. Manual on practical entomology in malaria. Part II. Geneva, WHO; 1995. WHO Offset Publication No. 13.

24. Lee KJ, Kim CB, Choi BJ, Park KH, Park JK. Analysis of vivax malaria cases in Gangwon-do (Province), Korea in the year 2000. Korean J Parasitol. 2001;39:301-6.

25. Bray RS, Garnham PCC. The life-cycle of primate malaria parasites. Br Med Bull. 1982;38:117-22.

26. Oh MD, Shin H, Shin D, Kim U, Lee S, Kim N, et al. Clinical features of vivax malaria. Am J Trop Med Hyg. 2001;65:143-6.

27. Jeffery GM, Young MD, Eyles DE. The treatment of Plasmodium falciparum infection with chloroquine, with a note on infectivity to mosquitoes of primaquine-and pyrimethamine-treated cases. Am J Hyg. 1956;64:1-11.

28. Nacher M, Silachamroon U, Singhasivanon P, Wilairatana P, Phumratanaprapin W, Fontanet A, et al. Risk factors for Plasmodium vivax gametocyte carriage in Thailand. Am J Trop Med Hyg. 2004;71:693-5.

29. Lee JS, Kho WG, Lee HW, Seo M, Lee WJ. Current status of vivax malaria among civilians in Korea. Korean J Parasitol. 1998;36:241-8.

30. Lee HI, Lee JS, Shin EH, Lee WJ, Kim YY, Lee KR. Malaria transmission potential by Anopheles sinensis in the Republic of Korea. Korean J Parasitol. 2001;39:185-92.

31. Kiszewski A, Mellinger A, Spielman A, Malaney P, Sachs SE, Sachs J. A global index representing the stability of malaria transmission. Am J Trop Med Hyg. 2004;70:486-98.

32. Park CG, Chwae YJ, Kim JI, Lee JH, Hur GM, Jeon BH, et al. Serologic responses of Korean soldiers serving in malaria-endemic areas during a recent outbreak of Plasmodium vivax. Am J Trop Med Hyg. 2000;62:720-5.

33. Tada MS, Marques RP, Mesquita E, Dalla Martha RC, Rodrigues JA, Costa JD, et al. Urban malaria in the Brazilian Western Amazon Region I: high prevalence of asymptomatic carriers in an urban riverside district is associated with a high level of clinical malaria. Mem Inst Oswaldo Cruz. 2007;102:263-9.

34. Eliades MJ, Shah S, Nguyen-Dinh P, Newman RD, Barber AM, Roberts JM, et al. Malaria Surveillance United States, 2003. Centers for Disease Control and Prevention. Morb Mortal Wkly Rep. 2005;54(SS02):25-39.

35. Tang JS, Chen CL, Ko WC, Chuang CC. Imported malaria in southern Taiwan from 1991 to 2002: a single hospital's experience. Kaohsiung J Med Sci. 2003;19:398-404. 